\title{
Criminologie
}

\section{Les victimes sans crimes : le traitement pénal des accidents de travail}

\section{Katherine Lippel}

Volume 21, numéro 1, 1988

Entre les mailles de la loi : pollution, accident de travail, faute professionnelle

URI : https://id.erudit.org/iderudit/017257ar

DOI : https://doi.org/10.7202/017257ar

Aller au sommaire du numéro

\section{Éditeur(s)}

Les Presses de l'Université de Montréal

ISSN

0316-0041 (imprimé)

1492-1367 (numérique)

Découvrir la revue

Citer cet article

Lippel, K. (1988). Les victimes sans crimes : le traitement pénal des accidents de travail. Criminologie, 21(1), 35-56. https://doi.org/10.7202/017257ar
Résumé de l'article

More people die in Canada every year from work related accidents and illnesses than as a result of murder. More work days are lost to work accidents and illnesses than to strikes and lockouts. Yet the illegalities committed in the field of health and safety in the workplace are, for all intents and purposes, left unnoticed by the criminal justice system.

This article addresses the use of penal and criminal law in Quebec against employers who have violated health and safety legislation and the Criminal Code.

The first part examines the historical, political and social reasons why deaths and injuries in the work place are perceived as normal and inevitable, rather than as aberra -tions often of a criminal nature.

The second part examines the application of statutory legislation in Quebec, particularly the Act Respecting Occupational Health and Safety (R.S.Q. c. S-2.1). The nature of the offences therein provided for is studied, as well as the attitude of the judiciary and the Quebec Health and Safety Commission (C.S.S.T.) towards their application.

In the final section the relevant provisions of the Criminal Code are studied, in the light of the rare examples from case law where criminal negligence charges have been laid.

The article concludes with the assertion that a change of attitude both on the part of the state and on the part of public opinion is necessary if we wish that health and safety in the workplace be taken seriously by employers.
Ce document est protégé par la loi sur le droit d'auteur. L'utilisation des services d’Érudit (y compris la reproduction) est assujettie à sa politique d'utilisation que vous pouvez consulter en ligne.

https://apropos.erudit.org/fr/usagers/politique-dutilisation/ 


\section{LES VICTIMES SANS CRIMES: LE TRAITEMENT PÉNAL DES ACCIDENTS DU TRAVAIL Katherine Lippel *}

More people die in Canada every year from work related accidents and illnesses than as a result of murder. More work days are lost to work accidents and illnesses than to strikes and lockouts. Yet the illegalities committed in the field of health and safety in the workplace are, for all intents and purposes, left unnoticed by the criminal justice system.

This article addresses the use of penal and criminal law in Quebec against employers who have violated health and safety legislation and the Criminal Code.

The first part examines the historical, political and social reasons why deaths and injuries in the work place are perceived as normal and inevitable, rather than as aberra tions often of a criminal nature.

The second part examines the application of statutory legislation in Quebec, particularly the Act Respecting Occupational Health and Safety (R.S.Q.C.S-2.I). The nature of the offences therein provided for is studied, as well as the attitude of the judiciary and the Quebec Health and Safety Commission (C.S.S.T.) towards their application.

In the final section the relevant provisions of the Criminal Code are studied, in the light of the rare examples from case law where criminal negligence charges have been laid.

The article concludes with the assertion that a change of attitude both on the part of the state and on the part of public opinion is necessary if we wish that health and safety in the workplace be taken seriously by employers.

\section{INTRODUCTION}

On pourrait s'étonner devant l'idée de l'employeur-criminel. Même ceux qui sont sensibilisés aux white-collar crimes, au crime

* Avocate et professeure au Département des sciences juridiques, Université du Québec à Montréal, C.P. 8888, succ. « A», Montréal, Québec H3C 3P8.

Criminologie, $X X I, 1$ 
corporatif, envisagent davantage les situations de fraude et d'évasion fiscale, mais non pas les crimes contre la personne. Cet étonnement est le produit d'un cercle vicieux; les criminologues, les juristes et même la population en général, ont de la difficulté à donner le qualificatif de «criminel» aux gestes des administrateurs et directeurs de compagnies qui prennent des risques calculés et comptabilisés avec la vie de leurs employés. De ce fait, les poursuites criminelles sont extrêmement rares. Devant l'absence de poursuites, l'opinion publique se confirme à l'effet que les blessures et décès au travail sont des événements normaux, inévitables, des risques inhérents à la société moderne.

Pourtant, plus de journées de travail sont perdues à cause d'accidents et de maladies du travail qu'en vertu des grèves et lock-outl.

Allô Police ne s'attarde que rarement aux accidents du travail mortels, on dirait qu'un tel décès n'est pas suffisamment sensationnel. Pourtant, au début du siècle les décès au travail étaient effectivement objets de scandale: l'opinion publique n'y trouvait rien de normal'2. Il y a lieu de s'interroger sur les motifs de ce glissement, par lequel les morts au travail cessent de faire les manchettes et ainsi cessent de préoccuper l'opinion publique.

\section{LA NORMALISATION DES ACCIDENTS ET MALADIES AU TRAVAIL}

Pourquoi les décès dûs à des erreurs grossières du contremaitre ou des employeurs sont-ils qualifiés, dans les yeux de la population et de la justice, d' «accidents» alors que le même type d'erreur grossière d'un conducteur d'automobile ou d'un citoyen ordinaire constituerait de la négligence criminelle? Afin de répondre à cette question il faut regarder, dans son contexte historique, le traitement des accidents du travail par les juges et le législateur ${ }^{3}$.

1. Au Québec, en 1981, cinq millions de jours-personne ont été perdus à cause de l'ensemble des accidents et maladies du travail, comparativement aux 1790420 jourspersonne perdus, la même année, suite à des grèves et lock-out. Voir Donna Mergler, «La santé au travail: analyse du modèle médjcal et nécessité d'une approche préventive», communication présentée au Colloque syndical international sur la santé, Montréal, le 10 novembre 1987. En Ontario, en 1978, 12277000 jours-personne furent perdus à cause d'accidents ou maladies du travail et 6895000 jours-personne perdus suite à des grèves. Statistiques citées par Katherine Swinton, «Enforcement of Occupational Health and Safety Legislation: the Role of the Internal Responsibility System», dans Kenneth P. Swan et Katherine E. Swinton (édit.), Studies in Labour Law, Toronto, Butterworths, 1983 , pp. $143-175$, a la p. 144.

2. C. Lipton, The Trade Union Movement of Canada 1827-1959, Toronto, N.C. Press, 1973, p. 54.

3. Pour une analyse détaillée de l'ensemble de cette question voir : Katherine Lippel, le Droit des accidentés du travail à une indemnité; analyse historique et critique, Éditions Thémis, Montréal, 1986. 
Jusqu'en 1909, au Québec, l'ensemble des demandes d'indemnisation pour des accidents du travail était régi par les articles 1053 à 1056 du Code civil du Bas-Canada. Afin de recevoir un dédommagement quelconque, le travailleur, ou ses héritiers en cas de décès, devaient démontrer, devant la Cour supérieure siégeant avec jury, que les dommages (blessures ou décès) subis à cause de l'accident étaient dus en tout ou en partie à la faute civile de l'employeur ou à celle d'un de ces employés. Dans le cadre de chaque procès, le juge et le jury cherchaient à déterminer la cause de l'accident, enquêtaient sur les pratiques de l'employeur et sur le mode de fonctionnement de l'usine. Il n'était pas rare de trouver des jugements dans lesquels les juges dénonçaient la recherche de profits par les employeurs, qui faisaient fi de la santé et de la sécurité des ouvriers ${ }^{4}$. L'appareil judiciaire s'intéressait au fonctionnement interne de l'usine et allait parfois jusqu'à s'immiscer dans les pratiques de production, tout en concluant que le mode de rémunération (tel le paiement à la pièce), les heures prolongées de travail ou même le manque de surveillance des ouvriers constituaient des fautes civiles engageant la responsabilité de l'employeur envers l'ouvrier victime d'un accident.

Malgré l'attitude plutôt ouverte des juges à l'égard des réclamations des ouvriers, la majorité des travailleurs demeuraient sans indemnisation, soit parce qu'ils n'avaient pas les moyens de poursuivre l'employeur en justice, soit parce que les blessures subies étaient dues à un accident qu'on qualifiait d' «anonyme» n'étant imputable à personne ${ }^{5}$. Les employeurs étaient également insatisfaits de la situation. Les condamnations par les tribunaux civils affectaient la prévisibilité des coûts de production et menaçaient parfois la rentabilité de leurs opérations.

Une première loi sur les accidents du travail fut adoptée en 19096, et par son adoption le législateur québécois reconnaissait pour la première fois qu'un accident du travail représentait un risque social. Cette loi d'indemnisation prévoyait que l'accident, survenant par le fait ou à l'occasion du travail, donnait ouverture à une indemnisation représentant $50 \%$ de la perte du salaire du travailleur, sans que ce dernier ait à prouver la faute de l'employeur pour être indemnisé. En

4. Voir notamment les arrêts cités dans K. Lippel, le Droit des accidentés au travail à une indemnité, op. cit, pp. 23 et suiv.

5. Voir le Rapport de la Commission d'enquête sur les accidents du travail (Commission Globensky), Québec, 1908.

6. Loi concernant les responsabilités des accidents dont les ouvrier sont victimes dans leur travail et la réparation des dommages qui en résultent, S.Q. 1909, c. 66. 
échange, les travailleurs perdaient le droit de poursuivre l'employeur pour les dommages réellement subis?. La loi de 1909 ne s'appliquait qu'à quelques industries; si le travailleur auvrait dans une industrie visée, il devait nécessairement se prévaloir de la nouvelle législation pour être indemnisé. Les travailleurs qui n'étaient pas visés par la loi devaient avoir recours au Code civil du Bas-Canada.

Le processus d'instauration de ce système d'indemnisation ${ }^{8}$ se compléta en 1931 avec l'adoption de la Loi des accidents du travail, $1931^{9}$. Dorénavant, la majorité des travailleurs n'aura comme seul recours civil qu'une réclamation à la Commission des accidents du travail. Cette Commission aura juridiction exclusive pour percevoir une cotisation de l'ensemble des employeurs visés par la loi et pour adjuger l'ensemble des réclamations des travailleurs. Aucune audition publique ne sera tenue par cette Commission et la question de la faute patronale sera complètement évacuée du processus d'indemnisation. La réalité aujourd'hui est encore la même: la présence ou l'absence d'une faute patronale n'a aucune influence sur le processus d'indemnisation ${ }^{10}$ et les victimes de lésions professionnelles n'ont aucune possibilité de poursuivre leurs employeurs devant les tribunaux civils ${ }^{11}$.

L'ensemble de la législation portant sur l'indemnisation des accidents du travail se base sur la notion de risque professionnel. S'appuyant sur les propos de députés siégeant lors de l'adoption de la première loi française sur l'indemnisation des accidents du travail, Bruno Mattéi explique ainsi le rôle de cette notion de «risque professionnel» dans le processus de normalisation des accidents du travail:

7. Sauf dans les cas où le travailleur pouvait démontrer la faute inexcusable de l'employeur. Toutefois cette exception disparaîtra en 1928 avec l'adoption de la Loi relative aux accidents du travail, S.Q. 1928, c. 79.

8. Certains disent que ce régime d'indemnisation crée «la responsabilité sans faute». Ce terme peut induire en erreur. Ce n'est pas que les accidents ne sont plus dus aux fautes des employeurs, mais que l'ouvrier $n$ 'a plus à prouver la faute patronale pour avoir droit à une indemnité. L'indemnité payable au travailleur est réduite, justement pour permettre à ceux qui n'auraient pas pu prouver la faute patronale de recevoir quand même quelque chose. Il n'y a aucune raison de croire que le comportement des employeurs après 1909 était moins fautif que celui d'avant 1909; au contraire, vu que les tribunaux n'examinaient plus le comportement patronal, l'incitation à la prévention des accidents était réduite.

9. S.Q. $1930-1931$, c. 100.

10. Loi sur les accidents du travail et les maladies professionnelles, L.R.Q.c. 3.001 , art. 25.

11. Loi sur les accidents du travail et les maladies professionnelles, L.R.Q. c. 3.001, art. 438; ceci est vrai même dans l'hypothèse où l'employeur se serait rendu coupable de négligence criminelle. 
'Le risque professionnel, c'est le risque afférent à une profession déterminée, indépendamment de la faute des ouvriers et des patrons.' 'L'ouvrier, cela est incontestable, court bien plus de risques qu'il y a 50 ans... il est exposé aux accidents par des risques inévitables: je dirai par la fatalité du milieux ambiant' (paroles de députés); ce qu'a voulu faire la bourgeoisie c'est en somme légaliser, institutionnaliser la fatalité; exposer les ouvriers au danger, ce n'est pas une faute, mais une nécessité liée au travail dans un univers industrialisé. Il en résultera un double bénéfice pour la bourgeoisie: faire admettre que travailler est dangereux: nier ensuite qu'il puisse y avoir un lien entre les accidents du travail et l'organisation du travail celle-ci se présente dans son innocence, mise en place par le seul discours rationnel et universel de la science et des techniques 12 .'

Le processus décrit par Mattéi dans le contexte français trouve son correspondant dans les contextes québécois et canadien. La recherche des causes d'accidents de travail n'est plus du domaine des tribunaux. Les journaux ne font donc plus état de procès où l'organisation du travail est examinée et jugée. Après plus d'un demi-siècle de camouflage du phénomène, l'opinion publique est pour le moins indifférente au sujet des accidents du travail, elle est beaucoup plus mobilisée par les risques des «vrais» crimes, crimes commis par des gens qui correspondent aux stéréotypes véhiculés par les médias. Si même les instances civiles n'enquêtent pas publiquement sur les causes d'accidents et de décès au travail, il est compréhensible que l'opinion publique, et même l'appareil judiciaire, n'aient que très peu de sensibilité à l'idée qu'une mort au travail puisse être une mort criminelle. Le responsable du décès est souvent l'organisation du travail. Or, les individus responsables de cette organisation du travail sont protégés par une structure corporative ou sociale qui permet facilement d'éviter la question de la responsabilité réelle pour l'accident. Charles Reasons explique ainsi ce phénomène:

We have been taught to think of crime as involving an easily identifiable victim and offender. For example, if our spouse poisons us and we subsequently die, then we have been murdered by our spouse, who is liable to prosecution. If, however, our company causes us to be exposed to toxic substances, and we subsequently die from this exposure, the company is not criminally liable for our death and will at the most be cited for violations of health and safety regulations. For example, it has been revealed that asbestos companies continued to expose workers to that substance in spite of the fact that they had had evidence concerning its fatal effects for some 30 years. Such premeditated

12. Bruno Mattéi, «La normalisation des accidents du travail: l'invention du risque professionnel», (1976) 354, les Temps modernes 988, à la p. 998. 
behaviour undoubtedly led to thousands of deaths and disabilities. Nonetheless, asbestos companies are only liable in civil lawsuits $^{13}$.

Malgré ce climat peu propice à la poursuite criminelle ou pénale des employeurs responsables d'accidents ou de maladies du travail, on compte quand même un certain nombre de dispositifs légaux qui permettent de telles poursuites, contrairement à ce qui se passe en matière civile ${ }^{14}$.

La possibilité de poursuites pénales en vertu de la législation portant sur la santé et la sécurité au travail existe depuis 1885 et elle est toujours vivante de nos jours. Il est également possible de porter des accusations contre des employeurs en vertu du Code criminel. Nous examinerons maintenant ces deux réalités.

\section{LA RESPONSABILITÉ PÉNALE DES EMPLOYEURS: LES POURSUITES PÉNALES EN VERTU DE LA LÉGISLATION SUR LA SANTÉ ET LA SÉCURITÉ AU TRAVAIL}

Dès 1885, l'Acte de manufactures de Québec a prévu l'interdiction suivante:

Il n'est pas permis de tenir une manufacture de manière que la vie de qui que ce soit qui y est employé soit en danger, ou de façon que la santé de ceux qui y sont employés soient probablement en danger d'être permanemment [sic] compromise ${ }^{15}$.

Aujourd'hui la Loi sur la santé et la sécurité au travail prévoit une infraction similaire:

Quiconque, par action ou par omission, agit de manière à compromettre directement et sérieusement la santé, la sécurité ou l'intégrité physique d'un travailleur est passible sur poursuite sommaire, en outre du paiement des frais, d'une amende d'au moins $500 \$$ et d'au plus $1000 \$$ ou d'une peine d'emprisonnement d'au moins deux mois et d'au plus six mois, ou d'une amende d'au moins 5000 \$ et d'au plus $20000 \$$ s'il s'agit d'une corporation ${ }^{16}$.

Entre ces deux textes de loi la législation québécoise a reflété une gamme d'interdictions et d'infractions; le fait pour un employeur de

13. Charles E. Reasons, «Ideology, Law, Public Opinion and Workers' Health», dans Dale Gibson \& Janet Baldwin (edit.), Law in a Cynical Society, Carswell Legal Publications, Calgary, 1985, pp. 262-282, à la p. 278.

14. Voir note 11, supra.

15. Acte des manufactures de Québec, 1885, S.Q. c. 32, art. 3.

16. L.R.Q. c. S-2.1, art. 237. 
mettre en péril la vie ou la santé d'un travailleur a toujours été illégal, tout au moins en théorie ${ }^{17}$. En pratique, par contre, cette législation fut traditionnellement inefficace dans la protection de la santé des travailleurs et ceci pour plusieurs raisons.

Premièrement, les normes législatives de sécurité sont la plupart du temps tellement tolérantes qu'elles ne garantissent aucunement une sécurité effective pour les travailleurs. Deuxièmement, même lorsque ces normes relativement généreuses sont outrepassées, les ressources disponibles pour assurer leur application sont souvent défaillantes ou inexistantes. Troisièmement, dans les quelques cas où une poursuite pénale est effectivement intentée, la majorité des plaintes sont rejetées, soit à cause de l'inadéquation du texte juridique, soit à cause de l'attitude de la magistrature, souvent réticente à condamner un employeur. Finalement, dans les rares cas où l'employeur est condamné pour une infraction pénale, les sanctions prévues dans la législation sont souvent ridiculement faibles. Examinons maintenant chacun de ces quatre éléments qui, ensemble, contribuent à chasser du champ des préoccupations d'un grand nombre de personnes les questions relatives à la santé et à la sécurité au travail.

\section{A) L'ÉTABLISSEMENT DES NORMES}

Historiquement, la réglementation adoptée dans le domaine souffrait de beaucoup de lacunes. Souvent des substances dangereuses n'étaient tout simplement pas réglementées. Lorsqu'une réglementation existait, elle était habituellement tellement ambiguë qu'elle restait à toutes fins pratiques inapplicable ${ }^{18}$. Par ailleurs, lorsque des normes furent effectivement établies pour certains contaminants, le processus de fixation des niveaux permissibles du produit ne fournissait aucune garantie que la limite tolérée était effectivement sécuritaire. Ceci est encore vrai aujourd'hui.

Le lecteur non averti pourrait en effet croire que le législateur, lors de l'établissement des normes réglementaires adoptées dans le cadre de la législation sur la santé et la sécurité au travail, s'assurerait que le

17. Pour une analyse plus détaillée de l'évolution de ces législations voir: Katherine Lippel, «Droits des travailleurs québécois en matière de santé (1885-1981)», (19811982) 16, Revue juridique Thémis, 329-380. Pour une analyse de l'évolution législative ontarienne voir : Eric Tucker, «The Determination of Health and Safety Standards in Ontario 1860-1982 : From the Market to Politics to...?» (1984) 29, McGill Law Journal, 260-311.

18. Voir à ce sujet: Katherine Lippel, «Droit des travailleurs québécois en matière de sante», op. cit., pp. 354-359. 
taux d'exposition toléré soit fixé à un niveau qui ne nuirait pas à la santé de l'ensemble des travailleurs. Or, le mode d'établissement des normes de sécurité dans l'industrie n'est pas fondé sur le principe d'éliminer les riques, mais plutôt sur le désir de trouver un équilibre entre les coûts de l'élimination du risque et les coûts moyens pour la santé des travailleurs. On tolèrera les «concentrations de substances dans l'air auxquelles on croit que presque tous les travailleurs peuvent être exposés jour après jour sans effet néfaste ${ }^{19}$.

La plupart des normes industrielles québécoises s'inspirent des normes américaines ${ }^{20}$, bien que les normes québécoises soient souvent moins exigeantes pour l'industrie que celles adoptées aux États-Unis ${ }^{21}$. On constate, dans ce pays, une tendance à imposer un test de coûtsbénéfices avant l'adoption d'une norme, processus qui a pour effet de mettre un prix sur la santé des travailleurs 22 . Fait encore plus inquiétant, l'information scientifique concernant plusieurs substances dangereuses est souvent non disponible ou carrément camouflée ${ }^{23}$.

L'histoire de l'amiante nous fournit à ce sujet, un bel mais triste exemple 24 .

Dans un contexte où la déréglementation est à la mode aux ÉtatsUnis, et par ricochet au Canada et au Québec, l'exemple américain est loin d'être une garantie de sécurité. Tout au long de l'histoire de la réglementation en matière de la santé et de la sécurité au travail les

19. American Conference of Governmental Industrial Hygienists, TLV. Threshold Limit Values for Chemical and Physical Agents in the Workroom Environment with intended changes for 1978, Cincinnati, Ohio, 1978, cité par Donna Mergler et Luc Desnoyers, «La Recherche scientifique et la lutte pour la santé au travail» (1979), 3 . Cahiers du socialisme, 41-71 a la p. 46 (souligné dans le texte de Mergler et Desnoyers). Sur l'établissement des normes américaines, voir également: Harry J. Glasbeek et Susan Rowland, «Are Injuring and Killing at Work Crimes?» (1979), 17, Osgoode Hall Law Journal, 506-594 à 518 ss.

20. D. Mergler et L. Desnoyers, op. cit., p. 46.

21. Ibid., p. 55.

22. Le président Reagan a exigé que toute réglementation soit soumise à une analyse de coûts-bénéfices par l'Executive Order 12291 (1981) 46 F.R. 13 193. Certains croient, cependant, que la décision de la Cour suprême américaine dans American Textile Manufacturers Institute Inc. c. Donovan (1981) 452 U.S. 490 aura pour effet d'atténuer l'exigence présidentielle (voir, à ce sujet, Eric Tucker, op. cit., p. 263). À noter, toutefois, que Samuel A. Rea Jr., «Regulating Occupational Health and Safety», dans Donald D. Dewees (édit.), The Regulation of Quality: Products, Services, Workplaces and the Environment, Butterworths, Toronto, 1983, pp. 109-140, semble faire (p. 130) l'interprétation contraire de la position de la Cour suprême américaine.

23. Voir Samuel S. Epstein, The Politics of Cancer, Anchor Press, 1979.

24. Voir D. Mergler et L. Desnoyers, op. cit., pp. 56-65. Voir également Paul Brodeur, Outrageous Misconduct: The Asbestos Industry on Trial, Pantheon Books, New York, 1985. 
législateurs ont manifesté de la réticence à s'immiscer dans les affaires de l'industrie. On a assisté, depuis le début des années 80, dans la littérature, à une montée des revendications en faveur de la déréglementation de l'industrie, même en matière de santé et de sécurité des travailleurs. L'argument à l'appui de ces revendications soutient que les contraintes du marché suffisent pour inciter les employeurs à prévenir les lésions professionnelles: les travailleurs demanderont un salaire plus élevé pour un travail jugé dangereux ce qui, par crainte de ces nouvelles exigences salariales, incitera les employeurs à investir davantage dans la prévention des accidents 25 . Le caractère fallacieux d'un tel raisonnement, qui fait abstraction de la réalité des travailleurs, souvent mal informés des risques auxquels ils s'exposent et économiquement dépendants de leurs employeurs, fut bien démontré par plusieurs auteurs 26 .

Bien que la thèse de la déréglementation ne semble pas avoir influencé le législateur québécois à un point tel qu'il ait abrogé les normes existantes 27 , elle contribue sans doute à freiner l'évolution de la législation et à rendre encore plus embrouillées les pratiques en matière d'application des normes.

\section{B) L'APPLICATION DES NORMES}

Toute interdiction qui ne s'accompagne pas de mesures effectives visant à garantir son application risque fort de rester lettre morte. Or, historiquement, les inspecteurs chargés de vérifier si les normes étaient respectées par l'industrie furent peu nombreux ${ }^{28}$. En outre, on a pu également constater que le fait de congédier ou de muter des inspecteurs peut constituer, pour l'État, un moyen efficace et discret de se dérober à l'application des normes qu'il impose à l'industrie ${ }^{29}$.

25. Voir notamment, Samuel A. Rea jr., op. cit.

26. Voir notamment Harry J. Glasbeek et Susan Rowland, op. cit., ainsi que Eric Tucker, op. cit., et Steven Kelman, «Occupational Safety and Health Administration», dans James Q. Wilson (Edit.), The Politics of Regulation, Basic Books inc., New York, 1980, pp. 236-266.

27. Il existe une gamme de règlements portant sur la santé et la sécurité au travail, voir notamment le Règlement sur la qualité du milieu de travail, R.R.Q. 1981, c. S-2.1, r. 14 et r. 15. Il existe également une dizaine de règlements applicables à des industries particulières.

28. Voir Katherine Lippel, Droit des travailleurs québécois en matière de santé, op. cit., pp. $356 \mathrm{ss}$.

29. Harry J. Glasbeek et Susan Rowland, op. cit., illustrent bien ce phénomène, à la p. 517 de leur article: «... in Manitoba an extensive scheme of employer-employee joint committees, backed by a governmental inspectorate and standard-setting agency was set up. However, as soon as the government changed from the New Democratic Party to the Progressive Conservative Party, the inspectorate was diminished in number to such an extent that the scheme has been rendered impotent." 
Puisque, bien entendu, il ne figure dans aucune loi, ni dans aucun règlement, un tel expédient contribue à ce que la législation en matière de santé et de sécurité au travail demeure un instrument de légitimation de l'action gouvernementale dans ce champ sans pour autant créer d'obstacles réels au fonctionnement de l'industrie. En 1987, au Québec, 226 inspecteurs travaillaient pour la Commission de la santé et de la sécurité au travail (C.S.S.T.) ${ }^{30}$, alors que 448 inspecteurs cuvraient à titre d'agents de conservation de la faune ${ }^{31}$.

Au Québec, la C.S.S.T. est chargée à la fois de l'indemnisation des victimes d'accidents du travail (comprenant la fonction d'imposer des cotisations à l'ensemble des employeurs), de la promotion de la prévention des lésions professionnelles et de l'inspection de l'ensemble des établissements. Ce triple rôle n'est pas sans créer certaines ambiguïtés: d'un côté, l'organisme agit comme tutelle d'assurance pour les employeurs, de l'autre, il est censé surveiller les industries en vue de garantir le respect des normes de sécurité adoptées par le législateur. Bien que nous ne soyons pas en mesure de démontrer, comme l'a fait Charles Reasons, en Alberta ${ }^{32}$, que les inspecteurs québécois préviennent l'industrie avant l'inspection, les statistiques de la Commission de la santé et de la sécurité au travail elle-même nous laissent perplexes quant à l'attitude des inspecteurs québécois vis-à-vis de l'industrie.

Ainsi, en 1985, les inspecteurs de la C.S.S.T. ont effectué 36344 «visites» d'établissements ${ }^{33}$. Une certaine proportion de ces visites fut provoquée par des accidents graves, des plaintes ou l'exercice d'un droit de refus. Lors de ces 36344 visites, 35407 infractions furent constatées. De ces 35407 violations de la loi, 1534 furent sanctionnées par l'imposition d'une amende. Seules 393 des amendes imposées furent payées et pas plus que 746 poursuites furent intentées devant les tribunaux. En autres termes, $4 \%$ des violations constatées par les inspecteurs ont fait l'objet de l'émission d'un avis d'infraction et seulement $2 \%$ des violations constatées ont mené à une poursuite pénale

30. Information obtenue, en décembre 1987, d'un membre du Conseil d'administration de la C.S.S.T.

31. Information obtenue, en décembre 1987, du directeur général adjoint de la D.G.O.R. du service de la conservation de la faune.

32. Charles E. Reasons, Lois L. Ross, et Craig Paterson, Assault on the Worker: Occupational Health and Safety in Canada, Butterworths, Toronto, 1981, p. 213.

33. Cette statistique, ainsi que celles qui suivront, proviennent du Rapport annuel de 1985 de la C.S.S.T., p. 33. 
devant les tribunaux ${ }^{34}$. Dans un des rares cas où une poursuite pénale $a$ effectivement eu lieu, la C.S.S.T., après avoir prouvé plusieurs infractions différentes commises par le même employeur, a fait une requête pour rouvrir la preuve afin de substituer, à la preuve déjà produite, la déclaration qu'elle n'avait pas de preuve à offrir. L'accusé fut évidemment acquitté 35 .

Bien sûr, le nombre d'infractions constatées par les inspecteurs, dans les établissements du Québec, en 1985, ne représente qu'une partie des infractions effectivement commises, moins que $10 \%$ des établissements ayant reçu la visite d'un inspecteur ${ }^{36}$.

Si les policiers téléphonaient au voleur avant d'arriver sur le lieu du crime et renonçaient à une poursuite pénale devant les tribunaux dans $98 \%$ des cas où le suspect était pris en flagrant délit, l'opinion publique réagirait. Pourtant, c'est ce qui arrive actuellement dans le domaine de la santé au travail. Il ne s'agit pas ici de prôner une politique systématique de poursuite pénale chaque fois qu'une violation de la législation sur la santé et la sécurité au travail est constatée. Par contre, on ne peut pas faire abstraction du bilan de la C.S.S.T. ellemême: sur 36344 visites, on a constaté 35407 violations de la loi, faisant une moyenne de 0,97 infractions par établissement. Il est évident que les outils d'incitation à la prévention des lésions professionnelles ne produisent pas l'effet escompté. On ne peut que se demander si les employeurs ne prendraient pas davantage au sérieux cette législation si les sanctions qui y sont prévues étaient effectivement appliquées.

\section{C) LE TRAITEMENT JUDICIAIRE DES POURSUITES PÉNALES}

Historiquement, la jurisprudence publiée portant sur la législation pénale en matière de santé et de sécurité au travail était plutôt rare ${ }^{37}$. À

34. Une telle réticence à poursuivre les employeurs n'est pas particulière aux inspecteurs québécois. Voir Glasbeek et Rowland, op. cit., p. 520.

35. C.S.S.T. c. Investissement Bocker Inc., Tribunal du travail, 500-28-001251-826, D.T.E. 83 T-652.

36. Selon le rapport annuel de la C.S.S.T., de 1984, 183533 établissements étaient visés par son mandat et il n'y a pas de raisons de croire qu'il y a eu une diminution de ce nombre en 1985. Des 36344 visites, en 1985, seulement 14838 sont qualifiées par la C.S.S.T., dans son rapport annuel de 1985, comme étant des visites «initiales» (p. 33 du rapport). Il faut conclure que plusieurs établissements furent visités à plusieurs reprises. Le Québec ne semble pas se distinguer particulièrement sur cette question. Aux ÉtatsUnis il est estimé que l'établissement du travailleur moyen est visité une fois par dix ans. Voir Rea, op. cit., p. 131.

37. On peut postuler que cet état des choses réflétait une pénurie de plaintes pénales. Il est également possible que les pénalités étaient tellement minimes qu'une contestation ne valait pas l'investissement. De fait, jusqu'en 1968 l'amende minimum payable pour une infraction commise sous la Loi sur les établissements industriels et commerciaux était de $10 \$$ (S.R.Q. 1964, c. 150, art. 30). 
la lecture des rapports judiciaires, on pouvait rester avec l'impression qu'il s'agissait d'un secteur de droit invisible, bien qu'une décision occasionnelle permettait de déceler l'interprétation extrêmement restrictive que les tribunaux réservaient à cette législation ${ }^{38}$.

Avec l'adoption de la Loi sur la santé et la sécurité au travail ${ }^{39}$, le législateur a instauré un nouveau système de poursuites pénales. Dorénavant, le Tribunal du travail sera saisi des plaintes pénales portées en vertu de la loi ${ }^{40}$; les poursuites pourront être intentées par l'association accréditée, par la C.S.S.T. ou un de ses mandataires, ou par «tout intéressé»41. Les amendes perçues reviennent à la C.S.S.T.42.

Il n'est pas de notre propos de faire une analyse descriptive des termes et de l'application de l'ensemble des infractions prévues dans cette loi ${ }^{43}$. Par contre, nous nous attarderons sur les deux infractions principales définies aux articles 236 et 237 de la loi:

236. Quiconque contrevient à la présente loi ou aux règlements ou refuse de se conformer à une décision ou à un ordre rendu en vertu de la présente loi ou des règlements ou induit une personne à ne pas s'y conformer, commet une infraction et est passible, sur poursuite sommaire, en outre du paiement des frais, d'une amende d'au moins 200 \$ et d'au plus 500 \$ s'il s'agit d'un individu, et d'une amende d'au moins $500 \$$ et d'au plus $1000 \$$ s'il s'agit d'une corporation.

En cas de récidive, les amendes prévues par le premier alinéa sont portées à un minimum de $500 \$$ et un maximum de $1000 \$$ s'il s'agit d'un individu, et à un minimum de 1000 \$ et un maximum de $2000 \$$ s'il s'agit d'une corporation.

237. Quiconque, par action ou par omission, agit de manière à compromettre directement et sérieusement la santé, la sécurité ou l'intégrité physique d'un travailleur est passible, sur poursuite sommaire, en outre du paiement des frais, d'une amende d'au moins $500 \$$ et d'au plus $1000 \$$ ou d'une peine d'emprisonnement d'au moins deux mois et d'au plus six mois, ou des deux à la fois, s'il s'agit d'un individu, ou d'une amende d'au moins 5000 \$ et d'au plus $20000 \$$ s'il s'agit d'une corporation.

38. Voir notamment Chaput c. Duquette et la Cité de Montréal, (1918) 54 C.S. 399, arrêt portant sur la Loi sur les établissements industriels. Voir également $R$. c. Canadian Vickers Ltêe (1978) R.P. (C.A.Q.).

39. S.Q. 1979 c. 63.

40. Ibid., art. 244.

41. Ibid., art. 242.

42. Jbid., art. 246.

43. Pour une description détaillée des différentes infractions et de leur application par les tribunaux voir : Denis Bradet, Bernard Cliche, Martin Racine et France Thibault, Droit de la santé et de la sécurité au travail: la loi et la jurisprudence commentées, Les Éditions Yvon Blais inc., Cowansville, 1986. 
En cas de récidive, les amendes et les peines d'emprisonnement prévues par le premier alinéa sont portées à un minimum de $1000 \$$ et à un maximum de $2000 \$$ et à un minimum de quatre mois et à un maximum de douze mois s'il s'agit d'un individu, et à un minimum de $10000 \$$ et à un maximum de $50000 \$$ s'il s'agit d'une corporation.

La jurisprudence établit clairement qu'il s'agit de deux infractions de responsabilité stricte 44 et que, par conséquent, l'accusé peut soulever avec succès une défense de diligence raisonnable ${ }^{45}$.

En principe, toute infraction aux normes de sécurité prévues par les règlements sera poursuivie, si poursuite il y a, en vertu de l'article 236 , à moins que le non-respect des normes soit de nature à compromettre directement et sérieusement la santé, et la sécurité ou l'intégrité physique d'un travailleur, auquel cas une poursuite en vertu de l'art. 237 pourrait être intentée.

Il n'est pas nécessaire qu'un travailleur ait été effectivement blessé pour qu'une poursuite en vertu de l'article 237 puisse être intentée 46 . Toutefois, parmi les 14 arrêts publiés en Droit du travail Express où l'accusé fut condamné en vertu de l'article 237, cinq impliquaient un accident grave ${ }^{47}$, quatre impliquaient le décès d'un ou de plusieurs travailleurs ${ }^{48}$, quatre ne spécifiaient pas s'il y avait eu ou non un accident ${ }^{49}$ et seulement un spécifiait qu'il n'y avait pas eu de blessés, bien qu'il y avait eu une explosion importante ${ }^{50}$. Par contre,

44. Voir notamment Poudrier et Boulet c. C.S.S.T., Cour superieure 200-36-00000383, D.T.E. 83T-553, relativement à l'article 236. Quant à l'article 237, voir Moisan c. les Aliments Ault Limitée, Tribunal du travail 500-28-000330-811, D.T.E. 82T-16.

45. Voir notamment C.S.S.T. et Nantel c. Inspection sous-marine J.P.B. Ltée, Tribunal du travail, 500-28-000505-834, D.T.E. 85T-9.

46. Roireau c. Produits chimiques Expro inc., Tribunal du travail, 500-28-001133826 , D.T.E. 84 T- 306.

47. C.S.S.T. c. Contenants industriels Ltée, Tribunal du travail, 500-29-000695-863, D.T.E. 87T-484, C.S.S.T. c. Eddy, Tribunal du travail, 500-28-000628-842, D.T.E. 85T10, Dagobert Schnubel (C.S.S.T.) c. Mines Noranda Ltée, Tribunal du travail 500-28001579-820, D.T.E. 84T-55, C.S.S.T. c. G.T.E. Sylvania Canada Ltée, Tribunal du travail, 200-28-000207-82, D.T.E. 84T-878, Moisan c. Aliments Ault Ltée, Tribunal du travail, 500-28-000330-811, D.T.E. 82T-16.

48. Constructions du St. Laurent Ltée c. Bourdages, Cour supérieure, 200-36000135-84, D.T.E. 85T-105, C.S.S.T. c. Cie de Construction M.F.M. Ltée, Tribunal du travail, 200-28-000593-837, D.T.E. 84T-707, Roireau c. Ouellette, Tribunal du travail, 500-28-001112-820, D.T.E. 83T-462, C.S.S.T. c. Société d'électrolyse et de chimie Alcan Ltée, Tribunal du travail, 200-28-000137-81, D.T.E. 83T-803.

49. C.S.S.T. c. Consolidated Bathurst Ltée, Tribunal du travail, 200-29-000094-86, D.T.E. 86T-653, C.S.S.T. c. P.G. du Québec, Cour supérieure, 200-36-000181-85, D.T.E. 86T-79, C.S.S.T. c. Achille de la Chevrotière Ltée, Tribunal du travail, 500-28-001555846, D.T.E. 85T-571, Moisan c. A.S.L.K. Canada Ltée, Tribunal du travail, 200-28000025-830, D.T.E. 84T-233.

50. Roireau c. Produits chimiques Expro Inc., Tribunal du travail, 500-28-001133826, D.T.E. 84 T-306 
dans les onze décisions publiées où les accusés furent acquittés de l'infraction prévue à l'article 237, au moins sept faisaient état d'un accident grave et cinq d'entre elles impliquaient le décès d'un ou plusieurs travailleurs ${ }^{51}$. Le lecteur reste avec l'impression que les poursuites en vertu de l'article 237 sont réservées à des événements extrêmement graves et que l'article sert davantage à punir le contrevenant, une fois les dommages causés, qu'à prévenir les accidents qui les occasionnent. Une telle ligne de conduite, de la part de la C.S.S.T., servirait semble-t-il à apaiser l'opinion publique, suite à un accident grave, tout en lui évitant d'intenter des poursuites lorsqu'une telle inaction pourrait passer inaperçue. Ceci expliquerait également pourquoi la majorité des causes publiées se rapportant à l'article 237 mènent à la condamnation du prévenu (14 sur 26), alors que la majorité des plaintes publiées, portées en vertu de l'article 236, sont rejetées (19 sur 29) ${ }^{52}$. Fait inquiétant, les décisions rapportées depuis 1986 démontrent une tendance très nette en faveur de l'acquittement pour les deux infractions art. 236, 8 acquittements et 1 condamnation depuis $1986^{53}$; art. 237,4 acquittements et 3

51. Sintra Inc. c. C.S.S.T, Cour supérieure, 200-36-000209-85, D.T.E. 86T-484 (décès), C.S.S.T. c. Construction Pagaro Inc., Tribunal du travail, 200-29-000095-86, D.T.E. 86T-535 (accident grave), Bovino c. M.W. Die Cutting Services inc., Tribunal du travail, D.T.E. 83T-222 (accident grave), C.S.S.T. c. Inter-Cité Construction Ltée, Tribunal du travail, 200-28-000127-826, D.T.E. 83T-223 (décès), C.S.S.T. c. Société d'électrolyse et de chimie Alcan Ltée, Tribunal du travail, 200-28-000136-819, D.T.E. 83 T804 (décès), Leclerc c. Maçonnerie Gagnon inc., Tribunal du travail, 500-28000714-816, D.T.E. 82T-389 (płusieurs décès), C.S.S.T. c. Industries Abex Ltée, Tribunal du travail, 500-28-000761-817, D.T.E. 82T-559 (plusieurs décès).

52. Il est évident qu'on ne peut pas se baser sur les arrêts publiés pour conclure nécessairement que l'ensemble des poursuites intentées suivent la même configuration. Les arrêts choisis pour fins de publication peuvent refléter davantage les partis pris de l'éditeur juridique que ceux des tribunaux eux-mêmes. Afin de confirmer les hypothèses soulevées ici, il serait souhaitable qu'une analyse de l'ensemble des décisions non publiées du Tribunal du travail soit effectuée. De plus, le chercheur devrait tenir compte des plaidoyers de culpabilité dans certains dossiers, information qui n'est jamais révélée dans les rapports judiciaires.

53. C.S.S.T. c. Construction Atlas Inc., Tribunal du travail, 500-29-000767-861, D.T.E. 87T-562, C.S.S.T. c. Communauté urbaine de Montréal, Cour supérieure, 500-36000649-866, D.T.E. 87T-782, C.S.S.T. c. Construction Lampron inc., Tribunal du travail, 200-29-000061-87, D.T.E. 87T-826, C.S.S.T. c. P.G. du Québec, Tribunal du travail, $655-$ 27-001546-856, D.T.E. 86T-21, Syndicat des fonctionnaires provinciaux du Québec inc. c. P.G. du Québec, Cour supérieure, 200-36-000127-85, D.T.E. 86T-109, C.S.S.T. c. Arno Électrique Ltée, Cour supérieure, 110-36-000042-847, D.T.E. 86T-235, Syndicat international des travailleurs unis de l'automobile, de l'aérospatiale et de l'outillage d'Amérique (T.U.A.) Local 1163, c. Général Motors du Canada Ltée, Tribunal du travail, 500-29-001542-859, D.T.E. 86T-430, C.S.S.T. c. Zinc électrolytique du Canada Ltée, Tribunal du travail, 500-29-001000-858, D.T.E. 86T-431 (acquittés sous 236 L.S.S.T.), Sidbec-Dosco inc. c. C.S.S.T., Cour supérieure, 500-36-000827-850, D.T.E. 86T-654 (condamné sous 236 L.S.S.T.). 
condamnations depuis 1986, alors que de 1982 à 1985 il n'y a eu que 7 acquittements en tout ${ }^{54}$. Encore une fois ces tendances peuvent $n$ 'être que le reflet du choix des arrêtistes, mais il serait important qu'une étude soit effectuée pour vérifier un certain nombre d'hypothèses. Parmi celles-ci, on peut se demander si le Tribunal n'est-il plus réticent à accueillir une plainte lorsque ce n'est pas la C.S.S.T., mais le syndicat, qui est le plaignant ${ }^{55}$. On devrait également analyser le lien entre les dommages subis par travailleurs et l'attitude du Tribunal. Finalement, une analyse détaillée de l'ensemble des plaintes pénales permettrait de mieux évaluer le degré de clémence dont fait preuve le Tribunal lors du prononcé de la sentence.

Malheureusement, on ne dispose à l'heure actuelle que des bribes d'information sur ces questions. Ainsi, la lecture de la jurisprudence rapportée peut facilement nous laisser l'impression que les tribunaux et particulièrement la Cour supérieure, qui a juridiction en appel - privilégient une approche restrictive de la législation. Cette dernière a en effet clairement statué que la Couronne, lorsqu'elle est condamnée pour l'infraction prévue à l'article 237 , doit être assimilée à un individu et non pas à une corporation pour les fins de l'établissement de la sentence ${ }^{56}$. Il fut également jugé que l'infraction prévue à l'article 237 exigeait une preuve de négligence, le juge allant jusqu'à dire que «le poursuivant doit prouver hors de tout doute raisonnable l'existence d'une situation ayant normalement des conséquences immédiates dramatiques pour la santé, la sécurité et l'intégrité physique d'un travailleur ${ }^{57}$.» Malgré la preuve d'un manquement au Code de sécurité, et malgré la preuve du décès du travailleur, le juge a quand même

54. Syndicat du transport de Montréal (employés des services d'entretien) C.S.N. c. S.T.C.U.M., Tribunal du travail, 500-29-000727-865, D.T.E. 87T-410, Sintra Inc. c. C.S.S.T., Cour supérieure, 200-36-000209-85, D.T.E. 86T-484, C.S.S.T. c. Construction Pagaro Inc., Tribunal du travail, 200-29-000095-86, D.T.E. 86T-535, 99935 Canada Ltée c. C.S.S.T., Cour supérieure, 200-36-000015-86, D.T.E. 86T-608, (acquittés sous 237 L.S.S.T.), Consolidated Bathurst Ltée c. C.S.S.T., Cour supérieure, 410-36-000006-861, D.T.E. 87T-430, C.S.S.T. c. Contenants Industriels Ltée, Tribunal du travail, 500-29-000605-863, D.T.E. 87T-484, C.S.S.T. c. P.G. du Québec, Cour supérieure, 200-36-000181-85, D.T.E. 86T-79 (condamnés sous 237 L.S.S.T.).

55. Récemment, selon un porte parole du contentieux de la CSN, certains bureaux régionaux de la C.S.S.T., en refusant de porter plainte contre des employeurs, ont suggéré aux syndicats de le faire eux-mêmes. Il est à craindre qu'une telle attitude laisse sans recours les travailleurs non syndiqués et que même les plus grosses centrales syndicales n'aient pas les moyens de financer la gestion des plaintes pénales qui pourraient être portées par la C.S.S.T., surtout quand on sait que les amendes sont payables non pas au poursuivant mais à la C.S.S.T.

56. C.S.S.T. c. P.G. du Québec, Cour supérieure, 200-36-000181-85, D.T.E. 86T-79.

57. 99935 Canada Ltée, c. C.S.S.T., Cour supérieure, 200-36-000016-86, D.T.E. 86T-608. 
acquitté la compagnie. L'exigence de preuve qu'il fait au poursuivant semble dépasser celle que le Code criminel prescrit en matière de négligence criminelle.

Même la Cour d'appel du Québec semble maintenir une approche restrictive et ce malgré l'élargissement par la Cour suprême du Canada de la notion traditionnelle d'interprétation restrictive du droit pénal 58 . Ainsi, la Cour d'appel a décidé que le terme «construction» ne comprenait pas la rénovation, afin de conclure que le Code de sécurité pour les travaux de construction ne s'appliquait pas à un travail de rénovation ${ }^{59}$. Encore récemment, la Cour d'appel a refusé d'appliquer la Loi sur la santé et la sécurité au travail à un poste de police, en se basant sur une interprétation littérale aux dépens d'une interprétation téléologique de ce texte de loi 60 .

Bien sûr, d'autres jugements existent où il apparaît que d'autres juges, particulièrement ceux du Tribunal du travail, résistent à des arguments techniques par lesquels certains employeurs essaient de contourner la loi 61 .

Afin de faire un bilan sur l'attitude judiciaire actuelle face aux plaintes pénales, il faudrait faire l'étude systématique de l'ensemble des décisions, publiées et non publiées. À défaut d'une telle étude, nous devons nous contenter, pour l'instant, d'une vision impressionniste de cette réalité qui nous laisse, d'ailleurs, plus que songeuse quant à l'efficacité réelle des poursuites pénales.

\section{D) LES SANCTIONS IMPOSÉES AUX CONTREVENANTS}

Les sanctions prévues dans la Loi sur la santé et la sécurité au travail sont quand même plus sévères que celles prévues, jusqu'en 1968, par la Loi sur les établissements industriels et commerciaux 62. Une infraction qui coûtera la vie à un travailleur coûtera maintenant plus que $10,00 \$$ à l'employeur, si ce dernier est effectivement pour-

58. Voir notamment Ogg-Moss c. La Reine, (1984) 2 R.C.S. 173.

59. Lapointe c. Duquette Construction Ltée, Cour d'appel du Québec, 500-28001246-826, D.T.E. 84T-322. D'autres décisions ont conclu que la démolition n'était pas un travail de construction. Voir C.S.S.T. c. Construction Paval inc., Tribunal du travail, 500-28-000848-837, D.T.E. 84T-578.

60. Communauté urbaine de Montréal c. C.S.S.T., (1987) R.J.Q. 272 (C.A.Q.).

61. Voir notamment C.S.S.T. c. Consolidated Bathurst ltée, Tribunal du travail, 20029-000094-86, D.T.E. 86T-653, confirmé à D.T.E. 87T-430., C.S.S.T. c. G.T.E. Sylvania Canada ltée, Tribunal du travail, 200-28-000207-82, D.T.E. 84T-878, ainsi que plusieurs décisions citées dans Denis Bradet et al., op. cit., pp. 178 ss.

62. Voir la note 40 , supra. 
suivi et condamné. Par ailleurs, les sanctions prévues aux articles 236 et 237 de la Loi sur la santé et la sécurité au travail sont maintenant proportionnelles à la gravité de la faute ${ }^{63}$.

Toutefois, on peut sérieusement questionner l'efficacité du système actuel, tant à cause du nombre tout à fait minime de poursuites et de condamnations, qu'en raison des sanctions réellement imposées par les tribunaux. Dans l'ensemble de la jurisprudence étudiée, nous n'avons repéré aucune condamnation à l'emprisonnement. Les recherches dans d'autres juridictions confirment d'ailleurs cette tendance ${ }^{64}$.

Les tribunaux québécois ont, heureusement, accepté une interprétation élargie de la notion de récidive: en effet, il n'est pas nécessaire que le contrevenant viole la même disposition réglementaire pour que les sanctions aggravées, prévues en cas de récidive aux articles 236 et 237 , s'appliquent ${ }^{65}$. Ces tribunaux ont, par contre, également décidé comme nous l'avons déjà mentionné66 - que la Couronne est assimilée à un individu pour les fins de détermination de la sentence, ce qui en pratique se traduit par l'imposition d'une amende minimum de $500 \$$ pour une infraction à l'article 237.

Or, des études américaines ont démontré qu'il fallait imposer une amende automatique variant de $3500 \$$ à $6800 \$$, pour chaque accident, afin d'en réduire la fréquence de $10 \%{ }^{67}$. Bien entendu, on est actuellement très loin de cette réalité au Québec, où les amendes sont rarement imposées et, en plus, sont suffisamment basses pour que les employeurs puissent facilement les intégrer à leurs coûts de production. En effet, lorsque l'employeur doit choisir entre la possibilité d'une poursuite pénale ( $2 \%$ des infractions constatées) et l'installation coûteuse d'un équipement sécuritaire, l'option de la violation de la loi demeure la plus profitable.

63. Voir toutefois nos commentaires sur des articles analogues dans la Loi sur les établissements industriels et commerciaux, L.R.Q. 1977, c. E-15, dans K. Lippel, «Droit des travailleurs québécois en matière de santé», op. cit., p. 366.

64. À ce sujet, Reasons et al. font le commentaire suivant: «While all jurisdictions allowed for the potential imprisonment of an offender, no one was ever incarcerated. In fact, we have not been able to find any case in the history of Canadian occupational safety and health laws where conviction resulted in imprisonment. Of course this cannot be said for murder, manslaughter or common assault", dans Reasons, Ross et Paterson, op. cit., p. 205.

65. Consolidated Bathurst ltée, c. C.S.S.T., Cour supérieure, 410-36-000006-861, D.T.E. 87T-430.

66. Voir la note 56 , supra.

67. Voir Rea, op. cit., p. 121. Le même auteur, à la p. 131, constate que l'amende moyenne par établissement américain entre 1971 et 1975 était de l'ordre de 1,52\$. 
Aussi longtemps qu'un tel choix n'impliquera pas une certaine réprobation sociale et, surtout, des sanctions économiques importantes, les employeurs continueront à opter pour le non-respect de la loi. Une des manières de changer l'attitude du public et, par ricochet, celle des employeurs, serait l'application du droit criminel actuellement en vigueur aux comportements négligents de certaines industries. Il y a lieu maintenant d'explorer cette voie.

\section{LA RESPONSABILITÉ CRIMINELLE DES EMPLOYEURS: LES POURSUITES EN VERTU DU CODE CRIMINEL}

Plusieurs excellentes études se sont penchées sur la question des poursuites criminelles des employeurs responsables d'accidents et de maladies du travail68. Glasbeek et Rowland font l'étude détaillée d'un ensemble de dispositions du Code criminel qui semblent particulièrement appropriées à la poursuite criminelle dans le cadre des accidents du travail et des maladies professionnelles. Il s'agit notamment des articles 202 (négligence criminelle), 245 et 245.1 (voies de fait et voies de fait grave), 380 (violation criminelle de contrat), 231 (trappes susceptibles de causer des lésions corporelles), 387 (méfaits), 176 (nuisance publique) et $212 \mathrm{c}$ (meurtre) ${ }^{69}$. Bien que l'analyse qu'ils font de l'ensemble de ces infractions soit fort intéressante, nous nous limiterons ici à avancer quelques propos au sujet de la négligence criminelle, cadre juridique le plus souvent invoqué, actuellement, lors des poursuites criminelles contre des employeurs. Il n'est donc pas de notre intention de faire l'analyse juridique de cette infraction qui se trouve définie dans le Code crinimel dans les termes suivants ${ }^{70}$.

202. (1) Est coupable de négligence criminelle quiconque,

a) en faisant quelque chose, ou

b) en omettant de faire quelque chose qu'il est de son devoir d'accomplir, montre une insouciance déréglée ou téméraire à l'égard de la vie ou de la sécurité d'autrui.

68. Voir notamment Glasbeek et Rowland op. cit., Reasons, Ross et Paterson, $o p$. cit., et les articles de Dick Martin et Charles Reasons, «Workers and Managers», et «Ideology, Law, Public Opinion and Workers" Health», dans Gibson et Baldwin, Law in a Cynical Society, op. cit., pp. 259 ss.

69. Voir Glasbeec et Rowland, op. cit., pp. 523-577.

70. Outre l'article de Glasbeek et Rowland, op. cit., le lecteur peut consulter A. Stalker, «Criminal Negligence» (1982), 7, Queen's Law Journal, 274, P. Bourque, «Proof of the Cause of Death in a Prosecution for Criminal Negligence Causing Death» (1980), 22, Criminal Law Quarterly 334; et Colvin, «Recklessness and Criminal Negligence» (1982), 32, U.TLJ. 345. 
(2) Aux fins du présent article, l'expression «devoir» signifie une obligation imposée par la loi71.

Il est bien établi que les corporations peuvent faire l'objet de poursuites criminelles 72 . Toutefois, il ne suffit pas de démontrer la négligence criminelle du contremaître, il faut plutôt faire la preuve que l'alter ego de la corporation fut négligent. Cette exigence fournit souvent un écran suffisant pour justifier l'acquittement de l'accusé.

Une poursuite pour négligence criminelle peut être intentée soit lorsque la négligence mène à des lésions corporelles, soit en cas de décès de la victime. Par contre, dans les rares cas où des poursuites criminelles furent intentées, les accusations faisaient suite à des accidents mortels et à des recommandations de poursuite de la part des coroners. Ceci pourrait s'expliquer en raison du fait que les accidents qui ne causent pas la mort ne font pas l'objet d'enquêtes publique ou semi-publique. Lorsque ces accidents se produisent, on n'appelle pas la police; au mieux, on appelle l'ambulance et la C.S.S.T.

Nous avons réussi à identifier dix accidents distincts qui ont donné lieu à des poursuites en vertu du Code criminel ${ }^{73}$ et, dans les dix cas, les accusés furent acquittés. Les deux seuls jugements où les accusés furent condamnés ont été ensuite renversés en appel ${ }^{74}$. Actuellement, à notre connaissance, un seul dossier est encore devant les tribunaux, la Cour d'appel du Québec ayant ordonné, dans ce cas, qu'un nouveau procès ait lieu ${ }^{75}$.

71. Les articles 203 et 204 édictent les infractions reliés à la négligence criminelle, causant la mort (203), et causant des lésions corporelles (204).

72. Voir notamment Union Colliery Co. c. The Queen, (1900) 31 S.C.R. 81, ainsi que la discussion de la queston dans Glasbeek et Rowland, op. cit., pp. 581 ss.

73. Nous avons été grandement assisté, dans la rédaction de cette section, par les résultats d'une recherche inédite effectuée par trois étudiants du Programme des sciences juridiques de l'U.Q.A.M.: Manon Bolliger, Ėve Montpetit et Ronald Prégent, la Responsabilité civile, criminelle et pénale des employeurs dans les cas d'accidents de travail, décembre 1984. Les dix décisions sont: Dame Glenda Deer c. Dominion Bridge Company Limited (1967), R.L. 241, $R$. c. Canadian Liquid Air Limited et al. (1973), 20 C.R.N.S. 208, C.I.P. c. R. (1979), C.A. 411, R. c. Chagnon, (1981), R.L. 454, R. c. St. Lawrence Mechanical Constructors Ltd., (1974), R.D.T. 401, R. c. R.B.C. Construction, 550-01-000641-74 (C.S.P.), 500-10-000316-776 (C.A.Q.), R. c. La Compagnie Sicotte, 500-01-003619-761 (C.S.P.), 500-10-000312-791 (C.A.Q.), R. c. Syncrude, (1984), 28 Alta. L.R. (2d) 233, R. c. Canadian Copper Mines Ltd., Journal de Montréal du 17 février 1982, p. 25, P.G. du Québec c. Belmoral Mines Ltée (N.P.L.), (1978), R.J.Q.); Cour supérieure, 615-01-000291-824.

74. Il s'agit des arrêts R. c. C.I.P. Inc., et R.B.C.Construction inc. c. R., précités à la note 73.

75. P.G. du Québec c. Belmoral Mines Ltée (N.P.L.) (1987), R.J.Q. 290, (C.A.Q.). 
De tels résultats reflètent éloquemment la réticence de l'appareil judiciaire à stigmatiser un citoyen corporatif par une condamnation criminelle. Ces dix causes ne furent certainement pas des plus banales, ayant déjà passé le stade d'enquête du coroner ainsi que celui de l'évaluation par le substitut du procureur général. Notons, toutefois, qu'il est fréquent que les juges voient, dans les témoignages des représentants des compagnies, l'expression d'un souci pour la sécurité de leurs employés et se fondent sur de telles preuves pour prononcer l'acquittement $^{76}$. Or, un tel raisonnement soumis par un conducteur d'automobile négligent, mais qui, dans le passé s'est soucié d'éviter un accident, serait non avenu. Bref, la structure corporative, la complexité de la réalité industrielle et, surtout, l'attitude des tribunaux envers ce genre de cause, contribuent ensemble à créer une classe d'infractions qui est à toute fin pratique à l'abri d'une condamnation criminelle.

Les juges québécois et canadiens ne sont pas les seuls à manifester de la réticence devant la poursuite criminelle des employeurs. Un juge américain a récemment déclaré non avenue une poursuite criminelle intentée contre un producteur de thermomètres. Dans cette cause, il fut mis en preuve que l'employeur cachait aux inspecteurs du Occupational Health and Safety Administration une chambre complète dans laquelle la compagnie recyclait le mercure utilisé dans ces thermomètres. Le travail dans cette chambre violait toutes les normes régissant la manipulation de cette substance toxique, ce qui a provoqué l'empoisonnement d'un des travailleurs de la compagnie. En ordonnant le sursis des procédures, nonobstant le fait que le jury considérait la compagnie coupable des accusations auxquelles elle faisait face, le juge du procès a conclu qu'une telle poursuite criminelle contre une compagnie ne pouvait avoir lieu, compte tenu de la possibilité de la poursuivre en vertu des infractions statutaires créées par le Occupational Health and Safety Administration Act ${ }^{77}$. Un tel raisonnement ne pourrait pas s'appliquer au Québec: l'existence, par exemple, du Code de la sécurité routière ne constitue pas un obstacle à une poursuite, en cette matière, en vertu du Code criminel. Il semble, cependant, que l'attitude du juge américain soit partagée par plusieurs juges québécois.

En France, la situation semble n'être guère différente. En 1975, le juge d'instruction Patrice de Charette est saisi d'un dossier de mort au

76. Voir Glenda Deer c. Dominion Bridge Company Limited (1967), R.L. 241, et C.I.P. c. R., (1979), C.A. 411.

77. Voir Jonathan A. Bennett, «Judge protects Pymms from Punishment», dans The Guardian, New York, 25 novembre 1987, p. 5. 
travail. Après enquête, il ordonne la mise en détention provisoire du P.D.G. Chapron ${ }^{78}$. Ce fut le scandale. Le juge Charette fera l'objet de menaces de poursuites disciplinaires, une Association de défense des intérêts moraux du personnel d'encadrement se mobilisera afin de faire pression sur le ministère de la Justice et, enfin, 390 cadres manifesteront dans la salle des pas perdus afin de revendiquer la libération de leur patron. Par un renversement des choses, ce fut la détention du P.D.G. et non pas le décès du travailleur qui a dérangé l'ordre social.

Au Québec, il n'est pas inusité d'assister à l'emprisonnement de chefs syndicaux qui se sont refusés de respecter des lois spéciales ordonnant le retour au travail suite à une grève légale. On attend encore la manchette où on apprendra qu'un chef d'entreprise fut emprisonné pour le décès d'un de ses travailleurs.

\section{CONCLUSION}

Il est manifeste que la poursuite pénale ou criminelle, suite aux accidents et maladies du travail, n'est pas encore intégrée dans les mœurs juridiques du Québec et du Canada. Il semble également évident que les mécanismes mis en place par le législateur pour prévenir les lésions professionnelles sont inefficaces et que cette inefficacité fait des milliers de victimes chaque année.

Peut-on conclure, de ces constatations, que l'on devrait prôner une politique systématique de poursuites pénales dans le domaine des lésions professionnelles? Pas nécessairement. On vit dans une société où le vol de nourriture, même d'une valeur de cinq ou dix dollars, fait encore l'objet de poursuites en vertu du Code criminel, avec l'imposition d'amendes et de casiers judiciaires. Il serait souhaitable que ceux qui appliquent le droit dans ce secteur s'inspirent de la clémence manifestée par les inspecteurs dans le domaine de la santé et de la sécurité au travail et par les juges qui ont été appelés à se prononcer sur des accusations de négligence criminelle portées contre des compagnies. Par contre, il serait également souhaitable que l'on cesse d'appliquer un double standard, selon l'identité du contrevenant, au moment de décider de l'opportunité des poursuites criminelles.

Enfin, il serait en fait ironique que le nouveau projet de loi du ministre Crosbie, offrant à la victime d'un crime la possibilité d'intervenir dans le procès criminel afin d'obtenir un dédommagement de

78. Voir «Un mort au travail» (1976) 354, les Temps modernes, 1004. 
l'accusé ${ }^{79}$, puisse - si approuvé - devenir une incitation à la poursuite criminelle des employeurs. Car, comme nous le savons, s'il est vrai que les victimes d'accidents du travail ne peuvent pas poursuivre leurs employeurs en dommages, il n'en est pas moins qu'elles conservent le droit de le faire devant les instances criminelles. Or, si l'on considère que certaines victimes d'accident du travail reçoivent, avec le nouveau régime d'indemnisation des lésions professionnelles, moins que jamais auparavant ${ }^{80}$, il n'est pas impensable que l'éventuelle instauration des mécanismes de dédommagement proposés par le ministre Crosbie vienne les encourager à intenter des poursuites criminelles contre leurs patrons. Et à force de le faire, il est à souhaiter que les tribunaux, ainsi que l'opinion publique, finiront sans doute par reconnaître que les accidents du travail ne constituent pas des occurrences normales. Simples hypothèses, admettons-le, car pour l'instant on doit plutôt conclure, à l'instar de François Ewald, que «la mort au travail au sein de l'atelier ne trouble pas l'ordre public; elle en ferait plutôt partie ${ }^{81 » \text {. }}$

79. Projet de loi C-89, Loi modifiant le Code criminel (Victimes d'actes criminels), Ire lecture, 5 novembre 1987, 35-36 Éliz II, art. 6, proposant de modifier l'article 653 du Code criminel.

80. Voir Katherine Lippel, «L'insécurité du revenu des accidentés du travail: les nouveautés dans 1'indemnisation des lésions professionnelles», dans ${ }^{D}$ Bureau et P. Mackay (édit.), le Droit dans tous ses états, Wilson et Lafleur, Montréal, 1987, pp. 285-317. p. 972.

81. François Ewald, «Présentation» (1976), 354, les Temps modernes 970 ss, à la 